\title{
Structure and Spectroscopic Properties of Hydrocalcium Isocyanide Isomers: Plausible Astronomical Ca-bearing Molecules
}

\author{
Pilar Redondo (iD, Antonio Largo (iD), and Carmen Barrientos (iD \\ Departamento de Química Física y Química Inorgánica, Facultad de Ciencias, Universidad de Valladolid, E-47011 Valladolid, Spain; carmen.barrientos@uva.es \\ Received 2020 May 21; revised 2020 July 15; accepted 2020 July 17; published 2020 August 24
}

\begin{abstract}
Recently, the first Ca-bearing molecule discovered in space, calcium isocyanide, CaNC, has been detected in the IRC +10216 circumstellar envelope. Related to CaNC are closed-shell stable molecules of [C, Ca, H, N] composition. One of the isomers with this composition is hydrocalcium isocyanide, HCaNC, which belongs to the hydrometal isocyanide/cyanide group of compounds, among which hydromagnesium isocyanide, $\mathrm{HMgNC}$, is, to date, the only one detected in space. In this work, we have performed a study of the $[\mathrm{C}, \mathrm{Ca}, \mathrm{H}, \mathrm{N}]$ isomers that are systems of possible interstellar interest by using quantum theoretical methodologies. The analysis of the relative energies predicts three low-lying isomers: c-HCa-NC $\left({ }^{1} \mathrm{~A}^{\prime}\right)$, hydrocalcium isocyanide, $\mathrm{HCaNC}\left({ }^{1} \Sigma\right)$, and hydrocalcium cyanide, $\mathrm{HCaCN}\left({ }^{1} \Sigma\right)$. At the composite level, $\mathrm{HCaNC}\left({ }^{1} \Sigma\right)$ and $\mathrm{HCaCN}\left({ }^{1} \Sigma\right)$ were located 0.87 and $4.84 \mathrm{kcal} \mathrm{mol}^{-1}$, respectively, above the c-HCa-NC $\left({ }^{1} \Sigma\right)$ isomer. Interconversion processes between these isomers have been studied to analyze their stability. Isomer $\mathrm{HCaNC}$ is characterized as a transition state and thus directly isomerizes to the $\mathrm{c}-\mathrm{HCa}-\mathrm{NC}$ minimum. The isomerization process of $\mathrm{HCaCN} \rightarrow \mathrm{c}-\mathrm{HCa}-\mathrm{NC}$ shows a small barrier of about $0.76 \mathrm{kcal} \mathrm{mol}^{-1}(\sim 382 \mathrm{~K})$ above $\mathrm{HCaCN}$, at the highest level of theory employed in this work, suggesting that $\mathrm{HCaCN}$ could easily isomerize into the most stable isomer, c-HCa-NC. Our results predict two low-lying isomers, namely, c-HCa-NC $\left({ }^{1} \mathrm{~A}^{\prime}\right)$ and hydrocalcium cyanide, $\mathrm{HCaCN}\left({ }^{1} \Sigma\right)$, as possible candidates for experimental or radioastronomical detection. For these isomers, we provide predictions for their vibrational and rotational spectroscopic parameters that could aid in their eventual characterization in the laboratory or in space.
\end{abstract}

Unified Astronomy Thesaurus concepts: Astrochemistry (75); Interstellar medium (847); Molecular spectroscopy (2095)

\section{Introduction}

The first metal-bearing molecules detected in space were the metal halides $\mathrm{NaCl}, \mathrm{AlCl}, \mathrm{KCl}$, and, tentatively, AlF. They were identified in 1987 in the circumstellar envelope (CSE) of the carbon-rich mass-losing star IRC +10216 (Cernicharo \& Guélin 1987). However, one year before, the discovery of a new free radical in the envelope of the same carbon star IRC +10216 (Guélin et al. 1986) had been reported. Even though the most likely candidates for such radicals were HSiCC (or its isomer HCCSi) and HSCC, the line carrier was definitively identified as MgNC in 1993 (Guélin et al. 1993; Kawaguchi et al. 1993). Since then, several metal-bearing molecules have been identified in the interstellar medium (ISM) or CSE. The list of interstellar metal-bearing species includes, in addition to the early-detected $\mathrm{MgNC}$ molecule, other metal cyanides or isocyanides such as $\mathrm{NaCN}$ (Turner et al. 1994), MgCN (Ziurys et al. 1995), SiCN (Guélin et al. 2000), AlNC (Ziurys et al. 2002), SiNC (Guélin et al. 2004), KCN (Pulliam et al. 2010), FeCN (Zack et al. 2011), and CaNC (Cernicharo et al. 2019).

Calcium is an interesting solar and interstellar element. It has a relatively high solar abundance $\left(\log \varepsilon_{\mathrm{Ca}}=6.34 \pm 0.04\right.$ $(\sigma=0.04))$ compared to other heavy elements such as $\mathrm{Na}, \mathrm{P}$, or K (Asplund et al. 2009). However, in the ISM, calcium can be incorporated into dust grains, and it is found to be highly depleted. This behavior was also observed through highresolution optical absorption spectroscopy in the carbon star

Original content from this work may be used under the terms of the Creative Commons Attribution 4.0 licence. Any further distribution of this work must maintain attribution to the author(s) and the title of the work, journal citation and DOI.
IRC+10216 (Mauron \& Huggins 2010). According to this study, calcium is significantly depleted onto dust grains in the $\mathrm{CSE}$, and this is the dominant form returned to the ISM. The depletion pattern is roughly consistent with expectations of dust condensation in a carbon-rich envelope. Mauron \& Huggins (2010) also pointed out that, although the metals are depleted in the envelope, atomic metals in the form of neutral atoms and ions appear to be the major metal species in the gas phase. As such, they likely play a key role in the metal chemistry of the envelope.

Calcium isocyanide, $\mathrm{CaNC}$, the first $\mathrm{Ca}$-bearing molecule discovered in space, has been recently detected in the IRC +10216 CSE (Cernicharo et al. 2019), where other metal isocyanides have been previously found. Calcium isocyanide is an open-shell system, and it can easily react with other species present in the ISM or CSE. The reaction of a hydrogen atom with $\mathrm{CaCN}$ gives the closed-shell stable molecule of hydrocalcium isocyanide, HCaNC. Consequently, this molecule could be thought to be present in space.

$\mathrm{HCaNC}$ belongs to the hydrometal isocyanide/cyanide compounds group among which hydromagnesium isocyanide, $\mathrm{HMgNC}$, is, to date, the only one detected in space. HMgNC was identified in 2013 in the laboratory and in the carbon-rich evolved star IRC +10216 (Cabezas et al. 2013). The authors pointed out that despite the chemistry of hydromagnesium isocyanide being uncertain, the $U$ shape of the line profiles of $\mathrm{HMgNC}$ observed in IRC +10216 indicates that the three $\mathrm{Mg}$ bearing molecules detected in space $(\mathrm{MgNC}, \mathrm{MgCN}$, and $\mathrm{HMgNC}$ ) could share a common origin in the cold outer envelope. 
Calculations of rate coefficients for the synthesis of metalcontaining molecules in IRC +10216 (Petrie 1996; Dunbar \& Petrie 2002) suggested the radiative association reactions of metal cations $\left(\mathrm{M}^{+}\right)$with $\mathrm{HCN} / \mathrm{HNC}$, followed by the dissociative recombination of $\mathrm{HMCN}^{+} / \mathrm{HMNC}^{+}$with an electron. In this context, the need for further studies to clarify the role of metal-containing molecules in the chemical evolution of cold astrophysical environments was highlighted.

Besides this astrochemical interest, the study of metal isocyanide/cyanide compounds has attracted attention in different areas such as supramolecular chemistry, crystal engineering, and hydrogen storage. Besides, the cyanide group shows a quasi-isotropic charge distribution, and some metal cyanides $\mathrm{MCN}(\mathrm{M}=\mathrm{Na}$ and $\mathrm{K})$ are polytopic molecules, where the $\mathrm{M}^{+}$cation seems to orbit the $\mathrm{CN}^{-}$ligand. On the other hand, cyanogen $(\mathrm{CN})$ is the simplest molecule including carbon and nitrogen, essential elements in the chemistry of life.

In this paper, we report a state-of-the-art theoretical study of $[\mathrm{C}, \mathrm{Ca}, \mathrm{H}, \mathrm{N}]$ isomers using accurate quantum chemistry approaches. We will analyze the thermodynamic stability of the different isomers, but special attention will be given to the most plausible astronomical molecules, namely, hydrocalcium isocyanide $(\mathrm{HCaNC})$ and hydrocalcium cyanide $(\mathrm{HCaCN})$. In addition, to shed light on the stability of these molecules in space, we will carry out a detailed analysis of the interconversion processes between isomers. Our main goal is to provide predictions for their spectroscopic parameters, which could aid in their eventual characterization in the laboratory or in space.

\section{Computational Methods}

The $[\mathrm{C}, \mathrm{Ca}, \mathrm{H}, \mathrm{N}]$ isomers were studied using ab initio and density functional methods. Within the density functional theory (DFT), we have employed the B3LYP (Becke threeparameter Lee-Yang-Parr) functional (Becke 1988; Lee et al. 1988) and the double hybrid B2PLYP functional (Grimme 2006). The B2PLYP functional is based on a mixing of standard generalized gradient approximations for exchange by Becke and for correlation by Lee, Yang, and Parr with Hartree-Fock exchange and a perturbative second-order correlation part.

Different ab initio approaches were also applied, ranging from second-order Møller-Plesset (MP2) perturbation theory (Møller \& Plesset 1934) to coupled cluster methodologies. In this case, we use the coupled cluster with single and double excitations (CCSD) approach, including triple excitations through a perturbative treatment, or $\operatorname{CCSD}(\mathrm{T})$ (Raghavachari et al. 1989). In the correlated calculations, the valence electrons of the carbon and nitrogen atoms and the $3 \mathrm{~s}$ and $3 \mathrm{p}$ electrons of calcium were included.

For the DFT calculations, we have used Pople's triple-zeta 6-311++G(3df, 2p) basis set (Hehre et al. 1986), which includes both polarization and diffuse functions on heavy atoms and hydrogen. For ab initio computations, in addition to Pople's basis set, we have employed different Dunning's correlation-consistent basis sets (Dunning 1989; Woon \& Dunning 1993).

Harmonic vibrational frequencies have been computed on each fully optimized structure. Vibrational calculations allow us to compute the zero-point vibration (ZPV) corrections and also classify the structures as true minima (all of the frequencies are real) or transition state structures (one of the frequencies, and just one, is imaginary). To provide accurate information that could help in a possible experimental identification by IR spectroscopy, anharmonic corrections were estimated for the most stable isomers.

Anharmonic frequencies were computed at the $\operatorname{CCSD}(\mathrm{T}) /$ cc-pVTZ level of theory using the second-order perturbation treatment (VPT2; Mills 1972) within the context of the Watson Hamiltonian (Watson 1968). A full cubic force field (CFF) and semidiagonal quartic force constants have been included in the procedure. From the CFF calculations, we have computed vibration-rotation interaction constants.

In order to refine structural parameters and energies, a composite scheme has been applied. Composite extrapolation methods rely on the assumption that an estimated property can be approximated as a sum of additive contributions. The first systematic model chemistry of this type was Gaussian-1 (G1; Pople et al. 1989). In the present work, we have applied a composite procedure in a way similar to that we used in previous works (Redondo et al. 2016, 2019), taking the CCSD (T)/cc-pVTZ calculations as a starting point. Within this scheme, the estimated geometrical parameter or electronic energy denoted as $\mathrm{P}$ (comp) is obtained as a sum of three terms:

$$
P(\text { comp })=P(\mathrm{CBS})+\Delta P(\mathrm{CV})+\Delta P(\text { aug }) .
$$

The first term, $P(\mathrm{CBS})$, corresponds to the complete basis set (CBS) limit and takes into account basis-set truncation errors. It is computed from the $n^{-3}$ extrapolation equation (Helgaker et al. 1997) applied to the cases of $n=3$ (T) and $n=4(Q)$, using $\operatorname{CCSD}(\mathrm{T}) / \mathrm{cc}-\mathrm{pVTZ}$ and $\operatorname{CCSD}(\mathrm{T}) / \mathrm{cc}-\mathrm{pVQZ}$ data, respectively.

The second term, $\Delta P(\mathrm{CV})$, accounts for core-valence $(\mathrm{CV})$ effects. It is calculated by taking the difference between a calculation including all electrons and a frozen-core (fc) approach:

$$
\Delta P(\mathrm{CV})=P(\mathrm{CVTZ}, \text { all })-P(\mathrm{CVTZ}, \mathrm{fc})
$$

where $P(\mathrm{CVTZ}$, all) is the property (geometrical parameter or electronic energy) evaluated at the $\operatorname{CCSD}(\mathrm{T}) / \mathrm{cc}$-pCVTZ level correlating all electrons (full), and $P(\mathrm{CVTZ}, \mathrm{fc})$ corresponds to the property computed taking into account valence electrons for the carbon and nitrogen atoms and the $3 \mathrm{~s}$ and $3 \mathrm{p}$ electrons for calcium (frozen-core approach).

The last term, $\Delta P(\mathrm{aug})$, accounts for the effect of the inclusion of diffuse functions (aug) in the basis set. It is obtained from the following difference:

$$
\begin{aligned}
& \Delta P(\text { aug }) \\
& \quad=P(\operatorname{CCSD}(\mathrm{T}) / \text { aug-cc-pVTZ })-P(\operatorname{CCSD}(\mathrm{T}) / \mathrm{cc}-\mathrm{pVTZ}
\end{aligned}
$$

where the basis sets aug-cc-pVTZ for $\mathrm{C}, \mathrm{N}$, and $\mathrm{H}$ are the Dunning's correlation-consistent basis sets (Dunning 1989; Woon \& Dunning 1993) and for Ca the core-valence triple zeta (CVTZ) proposed by Iron et al. (2003).

In addition, in the computation of composite energies $(P=E)$, we have included the term corresponding to ZPV energy calculated at the $\operatorname{CCSD}(\mathrm{T}) / \mathrm{cc}-\mathrm{pVTZ}$ within the harmonic approach:

$$
E(\text { comp })=E(\mathrm{CBS})+\Delta E(\mathrm{CV})+\Delta E(\mathrm{aug})+\mathrm{ZPV} .
$$

For the most stable [C, $\mathrm{Ca}, \mathrm{H}, \mathrm{N}$,$] isomer, we have$ characterized the nature of the bonding through a topological 
analysis of the electronic density in the framework of Bader's quantum theory of atoms in molecules (QTAIM; Bader 1990).

Quantum calculations were carried out with the Gaussian 16 program package (Frisch et al. 2016) and the CFOUR Program (Matthews et al. 2020). The topological analysis of electron charge density was performed using the AIMAll package (Keith 2013) including standard thresholds.

\section{Results and Discussion}

\subsection{Structure and Energetics}

As pointed out in the introduction, to the best of our knowledge, there is no information, neither theoretical nor experimental, for the $[\mathrm{C}, \mathrm{Ca}, \mathrm{H}, \mathrm{N}]$ isomers, so we will carry out a preliminary study of all the possible rearrangements with the CHCaN molecular formula. Thus, we have considered open-chain (linear or bent) structures with the calcium atom located at one end of the chain or in a middle position, and cyclic (three-member ring) structures.

In Figure 1, we show the optimized structures for all of the $[\mathrm{C}, \mathrm{Ca}, \mathrm{H}, \mathrm{N}]$ isomers at the $\mathrm{CCSD}(\mathrm{T}) /$ aug-cc-pVTZ level. Relative energies computed at the B2PLYP/6-31++G(3df, $2 p)$ level of theory are also included. The geometrical parameters of the $\mathrm{HCCaN}$ and $\mathrm{HNCaC}$ isomers are estimated at the B2PLYP $/ 6-31++\mathrm{G}(3 \mathrm{df}, 2 \mathrm{p})$ level. For each isomer, we have considered both singlet and triplet electronic states. However, we have only included in Figure 1 the structures corresponding to the lowest-lying state of each isomer.

From Figure 1, we can observe that the three most stable isomers arise from the interaction between the calcium atom of hydrocalcium, $\mathrm{HCa}$, and the cyano radical, $\mathrm{CN}$. The interaction can occur in the line of the $\mathrm{C}-\mathrm{N}$ bond through either the carbon atom or the nitrogen atom, yielding hydrocalcium cyanide/ isocyanide, $\mathrm{HCaCN} / \mathrm{HCaNC}$, with open-chain structures or perpendicular to the $\mathrm{C}-\mathrm{N}$ bond, giving, in this case, the apparently three-member cyclic structure, c-HCa-NC. At the B2PLYP level, hydrocalcium isocyanide lies only $0.99 \mathrm{kcal} \mathrm{mol}^{-1}$ higher in energy than $\mathrm{c}-\mathrm{HCa}-\mathrm{NC}$, and the corresponding cyanide counterpart, hydrocalcium cyanide, $\mathrm{HCaCN}\left({ }^{1} \Sigma\right)$, is located $4.38 \mathrm{kcal} \mathrm{mol}^{-1}$ above c-HCa-NC. It should be pointed out that, at DFT levels of theory, both $\mathrm{HCaNC}$ and $\mathrm{HCaCN}$ are bent structures.

Following in energy are the six isomers that arise from the interaction, either linear or perpendicular, between the calcium atom and hydrogen cyanide/isocyanide $\mathrm{HCN} / \mathrm{HNC}$. The linear interaction can take place between calcium and the hydrogen atom, yielding $\mathrm{CaHCN} / \mathrm{CaHNC}$, or between $\mathrm{Ca}$ and the terminal $\mathrm{N}$ or $\mathrm{C}$ atom, giving $\mathrm{CaNCH} / \mathrm{CaCNH}$. The perpendicular interaction of calcium with $\mathrm{HCN}$ or $\mathrm{HNC}$ leads to the cyclic structures c-HC- $\mathrm{CaN}\left({ }^{3} \mathrm{~A}^{\prime}\right)$ and c-HN-CaC $\left({ }^{3} \mathrm{~A}^{\prime}\right)$.

$\mathrm{CaHCN}$ and $\mathrm{CaHNC}$ have a ${ }^{1} \Sigma$ ground state, and they are located 29.53 and $44.20 \mathrm{kcal} \mathrm{mol}^{-1}$, respectively, higher in energy than the lowest-lying structure c-HCa-NC $\left({ }^{1} \mathrm{~A}^{\prime}\right)$. The energetic gap between $\mathrm{CaHNC}$ and $\mathrm{CaHCN}\left(14.67 \mathrm{kcal} \mathrm{mol}^{-1}\right.$ ) is similar to the energy difference between $\mathrm{HCN}$ and $\mathrm{HNC}$ (14.99 $\mathrm{kcal} \mathrm{mol}^{-1}$ at the B2PLYP/6-311++G(3df, 2p) level of theory), indicating that these isomers can be seen as intermolecular complexes between the $\mathrm{Ca}$ atom and the $\mathrm{HCN}$ or $\mathrm{HNC}$ molecule. The high values found for the computed $\mathrm{Ca}-$ H distances (4.1815 $\AA$ and $3.7901 \AA$ for CaHCN and CaHNC, respectively) also confirm the intermolecular-complex nature of these compounds.
Finally, we have studied the two structures that are derived from the insertion of the carbon atom in the carbon-nitrogen bond of $\mathrm{HCN}$ or HNC, namely, $\mathrm{HCCaN}\left({ }^{3} \Delta\right)$ and $\mathrm{HNZCaC}$ $\left({ }^{3} \Delta\right)$. Both isomers have a triplet ground state and are, as expected, by far less stable than the global minimum c-HCa$\mathrm{NC}\left(163.52 \mathrm{kcal} \mathrm{mol}^{-1}\right.$ and $143.94 \mathrm{kcal} \mathrm{mol}^{-1}$, respectively).

Relative energies, with respect to the most stable structure for the $[\mathrm{C}, \mathrm{Ca}, \mathrm{H}, \mathrm{N}]$ isomers, computed at different levels of theory, are reported in Table 1 . In general, we observed a good agreement for the stability order of isomers calculated by the different methodologies used in the present study. As can be seen, regardless of the level of approach followed, the lowestenergy isomer was the cyclic structure $\mathrm{c}-\mathrm{HCa}-\mathrm{NC}$. In addition, when both the level of calculation is improved and the size of the basis set is increased, the c-HCa-NC isomer seems to be favored over $\mathrm{HCaNC}$ and $\mathrm{HCaCN}$. Thus, $\mathrm{HCaNC} / \mathrm{HCaCN}$ is located $0.25 / 4.56 \mathrm{kcal} \mathrm{mol}^{-1}$ higher in energy than c-HCa-NC at the B3LYP level, whereas at the $\operatorname{CCSD}(\mathrm{T})$ level the relative energies are $1.24 / 5.02 \mathrm{kcal} \mathrm{mol}^{-1}$. From Table 2, we can also observe that the relative energies calculated at the B2PLYP level are the closest to those calculated at the CCSD(T) level. Given the relative energies found for the different isomers, we will now focus on the three most stable ones, namely $\mathrm{HCaNC}$, $\mathrm{HCaCN}$, and $\mathrm{c}-\mathrm{HCa}-\mathrm{NC}$, which will be the most relevant from an experimental point of view.

Table 2 shows structural parameters as well as relative energies for the three most stable isomers. In order to display the effect of the different contributions on the relative energies, we have collected in the table the results obtained at four different coupled cluster levels of theory as well as those determined by the composite procedure. The table also includes information for the transition state structures corresponding to the isomerization processes between isomers. From Table 2, we observe a good agreement among the geometrical parameters computed with the different $\mathrm{CC}$ methodologies. Comparing the $\mathrm{C}-\mathrm{N}$ bond distances for the three isomers shown in Table 2, we observe that this distance in $\mathrm{HCaCN}$ with a cyanide arrangement is slightly shorter than that in cyanogen (1.1741 $\AA$ at CCSD(T)/aug-cc-pVTZ level). The opposite was found for the corresponding isomer with an isocyanide arrangement, $\mathrm{HCaNC}$, and for the cyclic isomer c-HCa-NC. Consequently, bonding to the $\mathrm{CN}$ unit by a calcium atom through the nitrogen atom slightly weakens the $\mathrm{C}-\mathrm{N}$ bond, whereas it is slightly reinforced if the bonding takes place through the carbon atom. This behavior was also observed in cyanides and isocyanides of first-row transition metals (Rayón et al. 2007) and in our previous studies on hydrides of zinc, iron, and titanium cyanide/isocyanide (Redondo et al. 2015, 2016, 2019).

All CC levels of theory employed including the composite formalism predict $\mathrm{c}-\mathrm{HCa}-\mathrm{NC}$ as the most stable isomer. However, the composite formalism places hydrocalcium isocyanide, $\mathrm{HCaNC}$, only $0.87 \mathrm{kcal} \mathrm{mol}^{-1}$ higher in energy than $\mathrm{c}-\mathrm{HCa}-\mathrm{NC}$. Within this formalism, the cyanide counterpart, $\mathrm{HCaCN}$, is located $4.84 \mathrm{kcal} \mathrm{mol}^{-1}$ above c-HCa-NC. It can be seen from Table 2 that the addition of triple excitations to the $\mathrm{CC}$ procedure stabilizes the cyclic isomer over both $\mathrm{HCaNC}$ and $\mathrm{HCaCN}$, whereas an increase in the size of the basis set leads to the opposite effect. In the same way, the inclusion of diffuse functions in the basis sets yields a slight destabilization of the $\mathrm{c}-\mathrm{HCa}-\mathrm{NC}$ structure compared to the open-chain $\mathrm{HCaNC}$ and $\mathrm{HCaCN}$ isomers. 

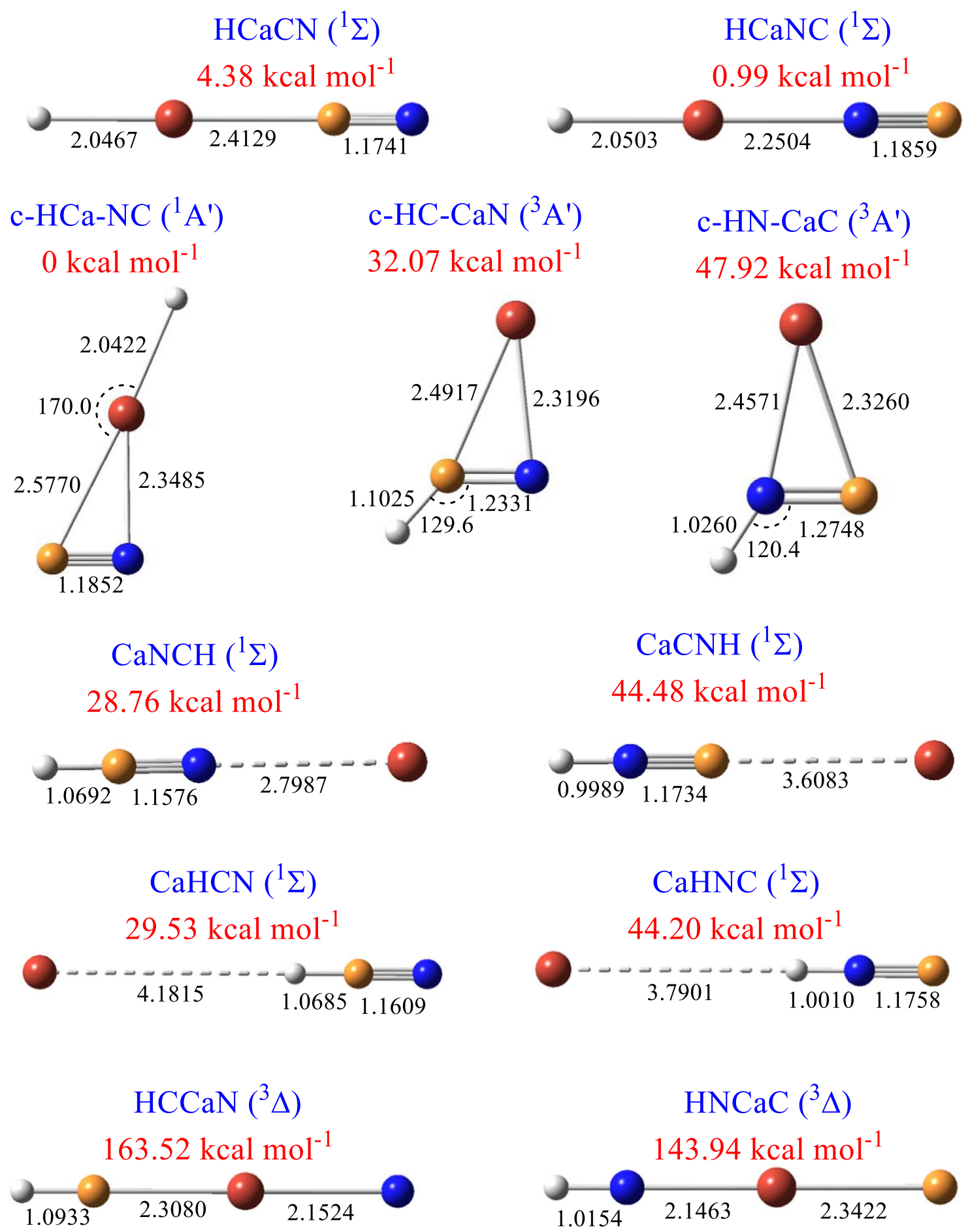

Figure 1. Optimized structures (distances in angstroms and angles in degrees) at the CCSD(T)/aug-cc-pVTZ level for the $[\mathrm{C}, \mathrm{Ca}, \mathrm{H}, \mathrm{N}]$ isomers. Relative energies (in $\mathrm{kcal} \mathrm{mol}^{-1}$ ) are computed at the B2PLYP/6-31++G(3df, 2p) level of theory. Optimized geometries for HCCaN and HNCaC are calculated at the B2PLYP/ 6-311++G(3df, 2p) level.

If we compare the energy of the $\mathrm{HCaNC}$ and $\mathrm{HCaCN}$ isomers shown in Table 2, we observe that, with the composite formalism, the isocyanide isomer is about $3 \mathrm{kcal} \mathrm{mol}^{-1}$ more stable than its cyanide counterpart. The stabilization of the isocyanide arrangement over the cyanide one was also observed in other hydrometal isocyanide/cyanide systems. For the first-row transition metals $\mathrm{Fe}, \mathrm{Ti}$, and $\mathrm{Zn}$, we observed that the stability of hydrometal isocyanide decreased with respect to hydrometal cyanide whenever going, in the periodic table, from early to late first-row transition metals. Thus, the energetic gap between the isocyanide and cyanide dispositions $\left(E_{\mathrm{HMNC}}-E_{\mathrm{HMCN}}, M=\mathrm{Fe}, \mathrm{Ti}, \mathrm{Zn}\right)$ is $4.07 \mathrm{kcal} \mathrm{mol}^{-1}$ for $\mathrm{Ti}$ (Redondo et al. 2019), $0.5 \mathrm{kcal} \mathrm{mol}^{-1}$ for Fe (Redondo et al. 2016), and $-5.13 \mathrm{kcal} \mathrm{mol}^{-1}$ for $\mathrm{Zn}$ (Redondo et al. 2015). This trend was also observed for first-row transition metal isocyanide/cyanide (Rayón et al. 2007).

The picture emerging from the most stable structure of the $[\mathrm{C}, \mathrm{Ca}, \mathrm{H}, \mathrm{N}]$ isomers, c-HCa-NC, corresponds to a three- 
Table 1

Relative Energies in $\mathrm{kcal} \mathrm{mol}^{-1}$ of the Different $[\mathrm{C}, \mathrm{Ca}, \mathrm{H}, \mathrm{N}]$ Isomers Calculated at Different Levels of Theory

\begin{tabular}{|c|c|c|c|c|c|c|}
\hline \multirow{2}{*}{ Isomer } & \multicolumn{6}{|c|}{ Level } \\
\hline & State & $\begin{array}{c}\text { B3LYP } / 6-311++G \\
\text { (3df, } 2 p)\end{array}$ & $\begin{array}{l}\text { B2PLYP } / 6-311++G \\
\quad(3 d f, 2 p)\end{array}$ & $\begin{aligned} \text { MP2 }= & \text { full } / 6-311++G \\
& (3 \mathrm{df}, 2 p)\end{aligned}$ & $\begin{array}{l}\text { CCSD/ } \\
\text { cc-pVTZ }\end{array}$ & $\begin{array}{c}\mathrm{CCSD}(\mathrm{T}) / \mathrm{aug}- \\
\text { cc-pVTZ }\end{array}$ \\
\hline $\mathrm{HCaCN}$ & ${ }^{1} \Sigma$ & 4.56 & 4.38 & 3.62 & 5.45 & 5.02 \\
\hline $\mathrm{HCaNC}$ & ${ }^{1} \Sigma$ & 0.21 & 0.99 & 2.32 & 1.66 & 1.40 \\
\hline $\mathrm{c}-\mathrm{HCa}-\mathrm{NC}$ & ${ }^{1} \mathrm{~A}^{\prime}$ & 0.00 & 0.00 & 0.00 & 0.00 & 0.00 \\
\hline $\mathrm{CaHCN}$ & ${ }^{1} \Sigma$ & 31.92 & 29.53 & 27.15 & 29.02 & 28.64 \\
\hline CaHNC & ${ }^{1} \Sigma$ & 45.13 & 44.20 & 44.59 & 43.09 & 42.87 \\
\hline $\mathrm{CaNCH}$ & ${ }^{1} \Sigma$ & 31.05 & 28.76 & 26.13 & 27.58 & 27.61 \\
\hline $\mathrm{CaCNH}$ & ${ }^{1} \Sigma$ & 45.34 & 44.48 & 44.36 & 42.81 & 43.05 \\
\hline c-HC-CaN & ${ }^{3} \mathrm{~A}^{\prime}$ & 29.57 & 32.07 & 36.23 & 35.40 & 35.74 \\
\hline c-HN-CaC & ${ }^{3} \mathrm{~A}^{\prime}$ & 44.39 & 47.92 & 52.72 & 49.61 & 50.73 \\
\hline $\mathrm{HCCaN}$ & ${ }^{3} \Delta$ & 155.68 & 163.52 & & & \\
\hline $\mathrm{HNCaC}$ & ${ }^{3} \Delta$ & 138.89 & 143.94 & & & \\
\hline
\end{tabular}

Note. ZPV energy corrections are included.

Table 2

Structural Parameters and Relative Energies of the [C, Ca, H, N] Isomers at Different Levels of Theory

\begin{tabular}{|c|c|c|c|c|c|c|c|}
\hline Level of Calculation & $\mathrm{CN}$ & $\mathrm{CaC}$ & $\mathrm{CaN}$ & $\mathrm{CaH}$ & $<\mathrm{NCCa}$ & $<\mathrm{CCaH}$ & $\Delta E$ \\
\hline \multicolumn{8}{|l|}{$\mathrm{c}-\mathrm{HCa}-\mathrm{NC}$} \\
\hline CCSD/cc-pVTZ & 1.1781 & 2.5848 & 2.3440 & 2.0336 & 65.0 & 170.3 & 0.00 \\
\hline $\operatorname{CCSD}(\mathrm{T}) / \mathrm{cc}-\mathrm{pVTZ}$ & 1.1855 & 2.5666 & 2.3528 & 2.0331 & 66.2 & 170.5 & 0.00 \\
\hline CCSD(T)/cc-pVQZ & 1.1812 & 2.5646 & 2.3396 & 2.0213 & 65.6 & 171.9 & 0.00 \\
\hline CCSD(T)/aug-cc-pVTZ & 1.1852 & 2.5770 & 2.3485 & 2.0422 & 65.5 & 170.0 & 0.00 \\
\hline Composite & 1.1758 & 2.5677 & 2.3236 & 2.0168 & 64.7 & 172.5 & 0.00 \\
\hline \multicolumn{8}{|l|}{$\mathrm{HCaNC}$} \\
\hline CCSD/cc-pVTZ & 1.1787 & $\cdots$ & 2.2478 & 2.0436 & & & 1.66 \\
\hline $\operatorname{CCSD}(\mathrm{T}) / \mathrm{cc}-\mathrm{pVTZ}$ & 1.1858 & $\cdots$ & 2.2481 & 2.0433 & & & 2.14 \\
\hline CCSD(T)/cc-pVQZ & 1.1819 & $\cdots$ & 2.2427 & 2.0322 & & & 1.80 \\
\hline CCSD(T)/aug-cc-pVTZ & 1.1859 & $\cdots$ & 2.2504 & 2.0503 & & & 1.42 \\
\hline Composite & 1.1771 & $\cdots$ & 2.2363 & 2.0268 & & & 0.87 \\
\hline \multicolumn{8}{|l|}{$\mathrm{HCaCN}$} \\
\hline CCSD/cc-pVTZ & 1.1661 & 2.4080 & $\cdots$ & 2.0397 & $\cdots$ & $\cdots$ & 5.45 \\
\hline $\operatorname{CCSD}(\mathrm{T}) / \mathrm{cc}-\mathrm{pVTZ}$ & 1.1737 & 2.4059 & $\cdots$ & 2.0397 & $\cdots$ & $\cdots$ & 5.37 \\
\hline CCSD(T)/cc-pVQZ & 1.1701 & 2.4012 & $\cdots$ & 2.0275 & $\cdots$ & $\cdots$ & 5.30 \\
\hline CCSD(T)/aug-cc-pVTZ & 1.1741 & 2.4129 & $\cdots$ & 2.0467 & $\cdots$ & $\cdots$ & 4.97 \\
\hline Composite & 1.1659 & 2.4011 & $\cdots$ & 2.0213 & $\cdots$ & $\cdots$ & 4.84 \\
\hline \multicolumn{8}{|l|}{ TS1 } \\
\hline CCSD/cc-pVTZ & 1.1686 & 2.3806 & 3.3049 & 2.0310 & 134.4 & 165.5 & 6.04 \\
\hline $\operatorname{CCSD}(\mathrm{T}) / \mathrm{cc}-\mathrm{pVTZ}$ & 1.1761 & 2.3795 & 3.3163 & 2.0305 & 134.9 & 164.8 & 5.92 \\
\hline CCSD(T)/cc-pVQZ & 1.1723 & 2.3730 & 3.2961 & 2.0174 & 133.9 & 163.7 & 5.86 \\
\hline $\operatorname{CCSD}(\mathrm{T}) / \mathrm{aug}$-cc-pVTZ & 1.1765 & 2.3862 & 3.3043 & 2.0403 & 133.0 & 169.4 & 5.69 \\
\hline Composite & 1.1679 & 2.3706 & 3.2541 & 2.0115 & 130.7 & 167.4 & 5.60 \\
\hline \multicolumn{8}{|l|}{$\mathrm{TS} 2$} \\
\hline CCSD(T)/aug-cc-pVTZ & 1.1862 & 3.3428 & 2.2490 & 2.0470 & $152.0^{\mathrm{a}}$ & $170.3^{b}$ & 1.42 \\
\hline
\end{tabular}

Notes. Bond distances are in angstroms and angles are in degrees. Relative energies are in kcal mol ${ }^{-1}$.

a $<\mathrm{CNCa}$.

b $<\mathrm{NCaH}$.

membered cyclic structure with the calcium atom bonded to hydrogen, carbon, and nitrogen (see Figure 1). However, in order to understand the nature of the bond interactions in this apparently cyclic structure, we have used the QTAIM theory. In Figure 2, we show the contour maps of the Laplacian of the electron density including the molecular graph corresponding to the $\mathrm{c}-\mathrm{HCa}-\mathrm{NC}$ isomer, and Table 3 resumes the local topological properties of the electronic charge density distribution calculated at the position of the bond critical points.

For the c-HCa-NC isomer, the molecular graph does not show any ring critical point, and only three bond critical points between $\mathrm{H}-\mathrm{Ca}, \mathrm{Ca}-\mathrm{N}$, and $\mathrm{N}-\mathrm{C}$ atoms were found. Therefore, this isomer can be described as a $\mathrm{HCa}$ unit $\pi$-bonded to the $\mathrm{CN}$ moiety through the calcium atom with an L-shaped structure. An inspection of the contour maps of the Laplacian of the electron density suggests that the $\mathrm{C}-\mathrm{N}$ bonds correspond to typical shared interactions, whereas both the $\mathrm{H}-\mathrm{Ca}$ and $\mathrm{Ca}-\mathrm{N}$ bonds show characteristics of closed-shell intermediate interactions. This global view was confirmed by the topological properties of the electronic densities collected in Table 3. The $\mathrm{C}-\mathrm{N}$ bond critical point is characterized by a large value of electron charge density, $\rho(r)$, and negative values of its 


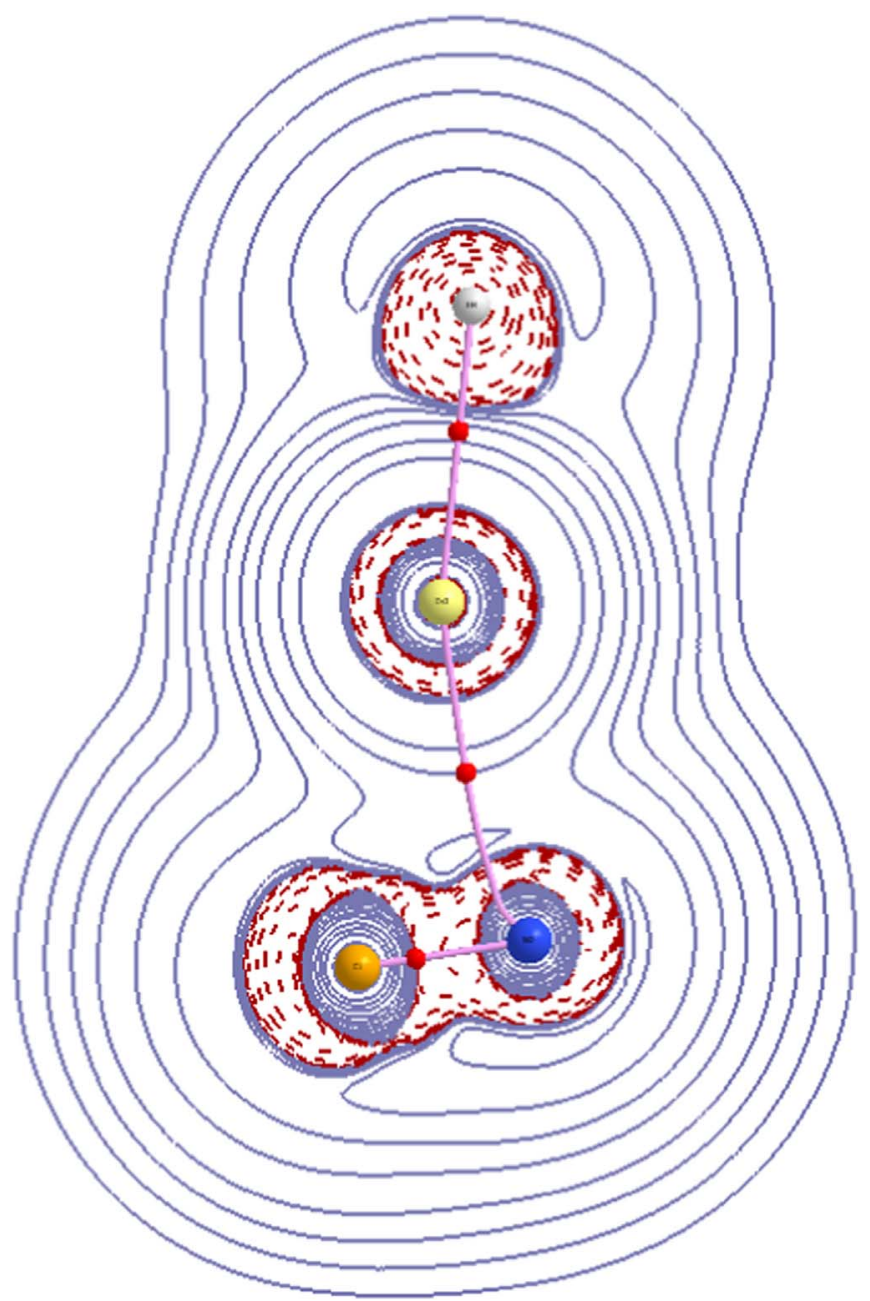

Figure 2. Contour maps of the Laplacian distribution of the electron density for the $\mathrm{c}-\mathrm{HCa}-\mathrm{NC}$ isomer. Small pink spheres represent bond critical points. Red dashed lines indicate regions of electronic charge concentration $\left(\nabla^{2} \rho(r)<0\right)$, and blue continuous lines denote regions of electronic charge depletion $\left(\nabla^{2} \rho(r)>0\right)$.

Table 3

Local Topological Properties (in au) of the Electronic Charge Density Distribution Calculated at the Position of the Bond Critical Points for the $\mathrm{c}-\mathrm{HCa}-\mathrm{NC}$ Isomer

\begin{tabular}{lcrcl}
\hline \hline Bond & $\rho(r)$ & $\nabla^{2} \rho(r)$ & $|V(r)| / G(r)$ & \multicolumn{1}{c}{$H(r)$} \\
\hline $\mathrm{Ca}-\mathrm{N}$ & 0.045 & 0.202 & 1.019 & -0.00098 \\
$\mathrm{C}-\mathrm{N}$ & 0.469 & -0.519 & 2.172 & -0.886 \\
$\mathrm{Ca}-\mathrm{H}$ & 0.050 & 0.120 & 1.205 & -0.0077 \\
\hline
\end{tabular}

Note. Local topological properties are in au. Columns are the electronic charge density $[\rho(r)]$, the Laplacian $\left[\nabla^{2} \rho(r)\right]$, the relationship between the local kinetic energy density $[G(r)]$ and the local potential energy density $[V(r)]$, and the total energy density $[H(r)]$.

Laplacian, $\nabla^{2} \rho(r)$. The large negative value for the total energy density $H(r)$ and the relationship between the local kinetic energy density $[G(r)]$ and the local potential energy density $[V$ $(r)]$, or $|V(r)| / G(r)$, greater than 2 confirm the essentially covalent nature of these interactions. On the other hand, both $\mathrm{H}-\mathrm{Ca}$ and $\mathrm{Ca}-\mathrm{N}$ bonds have small values of $\rho(r)$, positive values of $\nabla^{2} \rho(r)$, and small negative values of $H(r)$, and the value of the relationship $V(r) / G(r)$ is between 1 and 2 , showing

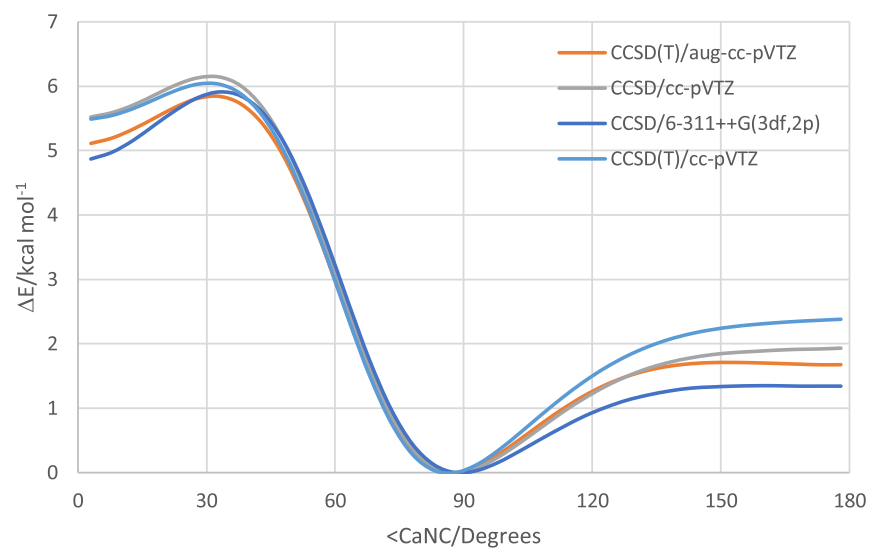

Figure 3. Graph of the scan obtained through partial optimizations at different $\angle \mathrm{CaNC}$ angles using several coupled cluster levels of theory.

an intermediate character (ionic with a certain degree of covalency).

To analyze the stability of the isomers, we have studied the possible interconversion reactions between isomers. A vibrational analysis for hydrocalcium isocyanide shows that this isomer has an imaginary frequency associated with the bending mode, at all CC levels of theory employed in the present work, except at the CCSD(T) level, when the aug-cc-pVTZ basis set is employed. Thus, this structure seems not to be a true minimum on the singlet potential energy surface (PES), but instead it should be considered as a transition state structure corresponding to the rotation movement of the $\mathrm{HCa}$ unit around the $\mathrm{C}-\mathrm{N}$ moiety. To confirm the transition state structure nature of the HCaNC conformer, we have carried out relaxed PES scans at different levels of theory. In Figure 3 we show the graph of the scan obtained through partial optimizations at different $\angle \mathrm{CaNC}$ angles using several CC levels of theory. In the scan, electronic energies (with no inclusion of $\mathrm{ZPV}$ corrections) are represented versus $\angle \mathrm{CaNC}$. When performing optimizations of the geometry, for fixed $\angle \mathrm{CaNC}$ angles ranging from $0^{\circ}$ to $180^{\circ}$, two minimum points are observed. The global minimum is located around $90^{\circ}$ and corresponds to the c-HCa$\mathrm{NC}$ isomer, whereas the local minimum, which is placed at $0^{\circ}$, represents the $\mathrm{HCaCN}$ structure. It should be noted that no minimum point was found for the $\angle \mathrm{CaNC}$ angle of $180^{\circ}$ corresponding to $\mathrm{HCaNC}$, except when the $\mathrm{CCSD}(\mathrm{T}) /$ aug-ccpVTZ (with no inclusion of ZPV corrections) level was used. At this level, as shown in Figure 3, we have located a transition state, TS2, associated with the interconversion between $\mathrm{HCaNC}$ and c-HCa-NC. However, as can be seen in Table 2, when ZPV corrections are included, TS2 and HCaNC have the same energy $\left(1.42 \mathrm{kcal} \mathrm{mol}^{-1}\right.$ at the $\operatorname{CCSD}(\mathrm{T}) /$ augcc-pVTZ level of theory), and consequently no barrier is found.

To confirm the presence of the barrier associated with the interconversion between the $\mathrm{HCaNC}$ and $\mathrm{c}-\mathrm{HCa}-\mathrm{NC}$ isomers, we have estimated the energy corresponding to the TS2 structure at the composite level considering the CCSD(T)/augcc-pVTZ optimized geometries. Within the composite formalism, we found that the energy barrier is practically null $\left(0.08 \mathrm{kcal} \mathrm{mol}^{-1}\right)$. At the highest level of theory used, the linear form is in fact a minimum on the PESs, but the flatness of the surface suggests that this has no practical significance; the structure will tend toward the more stable form. Only c-HCa$\mathrm{NC}$ and $\mathrm{HCaCN}$ isomers should eventually be considered as 


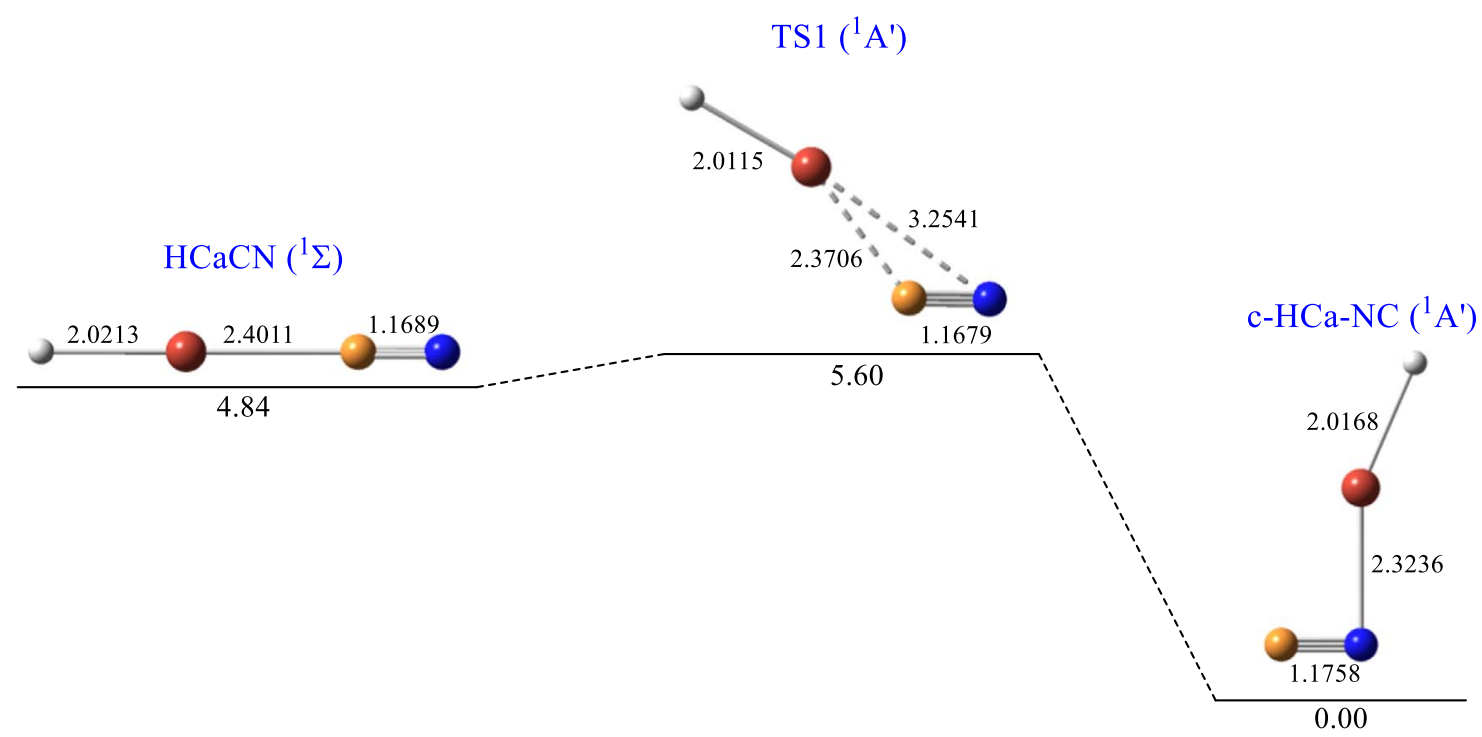

Figure 4. Energy profile for the isomerization process for $\mathrm{HCaCN} \rightarrow \mathrm{c}-\mathrm{HCa}-\mathrm{NC}$. Relative energies, in $\mathrm{kcal} \mathrm{mol}^{-1}$, are calculated with respect to c-HCa-NC at the composite procedure.

potential targets for experimental or radioastronomical detection.

In Figure 4 the energy profile for the isomerization process of $\mathrm{HCaCN} \rightarrow \mathrm{c}-\mathrm{HCa}-\mathrm{NC}$ computed with the composite procedure is shown. In addition, in Table 2, we have collected the relative energies corresponding to the transition state structure, TS1. The isomerization between $\mathrm{HCaCN}$ and $\mathrm{c}-\mathrm{HCa}-$ $\mathrm{NC}$ involves the transition state structure TS1 that is located only $0.76 \mathrm{kcal} \mathrm{mol}^{-1}(\sim 382 \mathrm{~K})$ above $\mathrm{HCaCN}$ at the composite level of theory. This small barrier suggests that $\mathrm{HCaCN}$ can easily interconvert into c-HCa-NC. Both isomers could be present in the cold regions of the ISM.

Finally, the stability is analyzed in terms of the dissociation energies. The following process is considered: $\mathrm{HCa}$ $(\mathrm{CN}) \rightarrow \mathrm{HCa}+\mathrm{CN}$. The results obtained for $\mathrm{HCaCN}$ and c-HCa-NC isomers at the $\operatorname{CCSD}(\mathrm{T}) /$ aug-cc-pVTZ level including ZPV corrections are $112.3 \mathrm{kcal} \mathrm{mol}^{-1}$ and $117.3 \mathrm{kcal} \mathrm{mol}^{-1}$, respectively. Therefore, both isomers are stable against fragmentation into $\mathrm{HCa}$ and $\mathrm{CN}$.

In addition, we have studied the stability of the isomers arising from the interaction of $\mathrm{Ca}$ with $\mathrm{HCN} / \mathrm{HNC}$, namely $\mathrm{CaHCN} / \mathrm{CaHNC}$ and $\mathrm{CaNCH} / \mathrm{CaCNH}$, relative to the formation of $\mathrm{HCN} / \mathrm{HNC}$ and a free Ca atom. At the CCSD(T)/augcc-pVTZ level of theory, including ZPV corrections, dissociation energies for $\mathrm{CaHCN} / \mathrm{CaHNC}$ are $0.38 / 0.75 \mathrm{kcal} \mathrm{mol}^{-1}$, and for $\mathrm{CaNCH} / \mathrm{CaCNH}, 1.41 / 0.56 \mathrm{kcal} \mathrm{mol}^{-1}$. These low values are consistent with the large bond distances found in these isomers between the $\mathrm{Ca}$ atom and the $\mathrm{HCN} / \mathrm{HNC}$ units (see Figure 1). Therefore, the $\mathrm{CaHCN} / \mathrm{CaHNC}$ and $\mathrm{CaNCH} /$ $\mathrm{CaCNH}$ isomers will be unstable with respect to dissociation to $\mathrm{HCN} / \mathrm{HNC}$ and a free $\mathrm{Ca}$ atom and confirm that the only isomers relevant from an experimental point of view will be $\mathrm{HCaCN}$ and $\mathrm{c}-\mathrm{HCa}-\mathrm{NC}$.

\subsection{Spectroscopic Parameters}

In Table 4, we show spectroscopic parameters relevant to the rotational spectroscopy, together with computed dipolemoment components along their principal inertial axes for the most stable isomers, namely c-HCa-NC and $\mathrm{HCaCN}$. Equilibrium rotational constants $\left(A_{\mathrm{e}}, B_{\mathrm{e}}\right.$, and $\left.C_{\mathrm{e}}\right)$ were computed from
Table 4

Rotational Spectroscopic Parameters for the $[\mathrm{Ca}, \mathrm{C}, \mathrm{H}, \mathrm{N}]$ Isomers (in $\mathrm{MHz}$ )

\begin{tabular}{lcc}
\hline \hline & c-HCa-NC & HCaCN \\
\hline$A_{\mathrm{e}}$ & 58838.6 & $\ldots$ \\
$B_{\mathrm{e}}$ & 5195.1 & 3089.7 \\
$C_{\mathrm{e}}$ & 4773.7 & $\ldots$ \\
$A_{0}$ & 59597.2 & $\ldots$ \\
$B_{0}$ & 5174.0 & 3149.2 \\
$C_{0}$ & 4746.0 & $\ldots$ \\
$\Delta_{\mathrm{J}} \times 10^{3}$ & 3.70360 & 0.935731 \\
$\Delta_{\mathrm{JK}} \times 10^{3}$ & 564.066 & -1.87146 \\
$\Delta_{\mathrm{K}} \times 10^{3}$ & 891.523 & 0.935731 \\
$\delta_{\mathrm{J}} \times 10^{3}$ & -0.482947 & 0.00000 \\
$\delta_{\mathrm{K}} \times 10^{3}$ & -0.326466 & 0.00000 \\
$\mu_{\mathrm{a}}$ & -1.33 & 3.42 \\
$\mu_{\mathrm{b}}$ & -0.22 & 0.00 \\
$\mu_{\mathrm{c}}$ & 0.00 & 0.00 \\
$\chi_{\mathrm{aa}}$ & 1.9028 & -5.5609 \\
$\chi_{\mathrm{bb}}-\chi_{\mathrm{cc}}$ & -6.1562 & 0.0000 \\
\hline
\end{tabular}

Notes. Equilibrium rotational constants $\left(A_{\mathrm{e}}, B_{\mathrm{e}}\right.$, and $\left.C_{\mathrm{e}}\right)$ and rotational constants for the ground vibrational state $\left(A_{0}, B_{0}\right.$, and $\left.C_{0}\right)$ are computed with the composite method. Centrifugal distortion parameters in the symmetrically reduced Hamiltonian $\left(D_{\mathrm{J}}, D_{\mathrm{JK}}, D_{\mathrm{K}}, d_{1}\right.$, and $\left.d_{2}\right)$ are included. The diagonal elements of the ${ }^{14} \mathrm{~N}$ nuclear quadrupole coupling tensor $\left(\chi_{\mathrm{aa}}, \chi_{\mathrm{bb}}, \chi_{\mathrm{cc}}\right)$ and dipole-moment components ( $\mu$ in Debye) are computed at the CCSD/cc-pVTZ level.

the bond distances computed with the composite level. The corresponding constants for the ground vibrational state were computed from vibration-rotation coupling constants and degeneracy factors for the vibrational modes from anharmonic vibrational frequencies at the CCSD(T)/cc-pVTZ level within the frozen-core approach. Centrifugal distortion parameters in the symmetrically reduced Hamiltonian $\left(D_{\mathrm{J}}, D_{\mathrm{JK}}, D_{\mathrm{K}}, d_{1}\right.$, and $d_{2}$ ) are also included. The diagonal elements of the ${ }^{14} \mathrm{~N}$ nuclear quadrupole coupling tensor $\left(\chi_{\mathrm{aa}}, \chi_{\mathrm{bb}}, \chi_{\mathrm{cc}}\right)$ and dipole-moment components ( $\mu$ in Debye) were computed at the CCSD/ccpVTZ level. The predicted dipolar moment of $\mathrm{HCaCN}$ (3.42 D) is very similar to that for $\mathrm{HMgNC}$ (3.49 D; Gronowski \& 
Table 5

Harmonic, $\omega$, and Anharmonic, $\nu$, Vibrational Frequencies $\left(\mathrm{cm}^{-1}\right)$ and IR Intensities $\left(\mathrm{km} \mathrm{mol}^{-1}\right)$ for the $\mathrm{c}-\mathrm{HCa}-\mathrm{NC}$ and $\mathrm{HCaCN}$ Isomers

\begin{tabular}{|c|c|c|c|c|c|c|c|c|c|}
\hline \multirow[b]{2}{*}{ Mode } & \multicolumn{4}{|c|}{ c-HCa-NC } & \multicolumn{5}{|c|}{$\mathrm{HCaCN}$} \\
\hline & $\omega$ & $I_{\text {har }}$ & $\nu$ & $I_{\text {anhar }}$ & Mode & $\omega$ & $I_{\text {har }}$ & $\nu$ & $I_{\text {anhar }}$ \\
\hline$a^{\prime \prime}$ & 116 & 504.9 & 120 & 484.2 & $\pi$ & 50 & 472.0 & 154 & 32.9 \\
\hline$a^{\prime}$ & 181 & 40.7 & 168 & 62.1 & $\sigma$ & 358 & 83.1 & 378 & 96.7 \\
\hline $\mathrm{a}^{\prime}$ & 388 & 96.1 & 387 & 21.1 & $\sigma$ & 1319 & 351.6 & 1293 & 322.2 \\
\hline $\mathrm{a}^{\prime}$ (H-Ca stretch) & 1324 & 354.3 & 1293 & 334.4 & $\sigma$ & 2144 & 32.5 & 2116 & 29.5 \\
\hline $\mathrm{a}^{\prime}(\mathrm{C}-\mathrm{N}$ stretch $)$ & 2044 & 24.9 & 2019 & 25.9 & $\ldots$ & $\ldots$ & $\ldots$ & $\ldots$ & $\ldots$ \\
\hline
\end{tabular}

Note. Harmonic and anharmonic vibrational frequencies are calculated at the $\operatorname{CSSD}(\mathrm{T}) / \mathrm{cc}-\mathrm{pVTZ}$ level.

Kolos 2013). The c-HCa-NC isomer has a lower dipolar moment, and its identification could be difficult in space.

Most of the molecules are detected in the ISM via their rotational signature, and thus the detection of a molecule in space depends on the availability of accurate predictions of rotational constants. In a benchmark study carried out by Alessandrini et al. (2018), the authors concluded that for the case of medium-sized molecules containing second-row elements, the use of a composite scheme, similar to that employed in the present work, was able to provide rotational constants that were sufficiently accurate to guide experiments. For the HMgNC isomer, using a VPT2 approach at the CCSD (T)/aug-cc-pVTZ level (Gronowski \& Kolos 2013), a value of the rotational constant, $B_{0}$, was predicted to be around $43 \mathrm{MHz}$ lower than the experimental value (Cabezas et al. 2013).

In order to help in an eventual detection of the c-HCa-NC and $\mathrm{HCaCN}$ isomers through IR spectroscopy, we give, in Table 5, harmonic, $\omega$, and anharmonic, $\nu$, vibrational frequencies and IR intensities for both isomers computed at the CCSD(T)/cc-pVTZ level. The results show that there is not any general scale factor between anharmonic and harmonic vibrational frequencies or intensities. Thus, in order to predict reliable IR spectra for these isomers, it is mandatory to include anharmonic contributions.

\section{Conclusions}

We have carried out a study of the $[\mathrm{C}, \mathrm{Ca}, \mathrm{H}, \mathrm{N}]$ isomers, which are systems of possible interstellar interest, by using quantum theoretical methodologies. We have considered openchain structures with the calcium atom located at one end of the chain or in a middle position, and cyclic (three-member ring) structures on both the singlet and triplet PES.

The three most stable isomers arise from the interaction between the calcium atom of hydrocalcium, $\mathrm{HCa}$, and the cyano radical, $\mathrm{CN}$. If the interaction occurs in the line of the $\mathrm{C}-$ $\mathrm{N}$ bond through either the carbon atom or the nitrogen atom, hydrocalcium cyanide/isocyanide isomers, $\mathrm{HCaCN}$ $\left({ }^{1} \Sigma\right) / \mathrm{HCaNC}\left({ }^{1} \Sigma\right)$, are obtained. When the interaction between $\mathrm{HCa}$ and $\mathrm{CN}$ takes place perpendicular to the $\mathrm{C}-\mathrm{N}$ bond, the apparently three-member cyclic structure c-HCa-NC is obtained.

At all levels of theory employed in this work, the lowestenergy isomer is the c-HCa-NC $\left({ }^{1} \mathrm{~A}^{\prime}\right)$ isomer, with the $\mathrm{HCaNC}$ $\left({ }^{1} \Sigma\right) / \mathrm{HCaCN}\left({ }^{1} \Sigma\right)$ isomers close in energy. A composite approach was applied to obtain accurate structures and relative energies for the most stable isomers. Within this procedure, hydrocalcium isocyanide $\mathrm{HCaNC}\left({ }^{1} \Sigma\right)$ and hydrocalcium cyanide $\mathrm{HCaCN}\left({ }^{1} \Sigma\right)$ were located 0.87 and $4.84 \mathrm{kcal} \mathrm{mol}^{-1}$, respectively, higher in energy than the c-HCa-NC $\left({ }^{1} \Sigma\right)$ isomer.
In addition, six isomers were obtained from the approach, either linear or perpendicular, between the $\mathrm{Ca}$ atom and $\mathrm{HCN}$ / HNC. These isomers were predicted to lie more than $20 \mathrm{kcal} \mathrm{mol}^{-1}$ above the lowest-lying isomer c-HCa-NC. Two structures that are derived from the insertion of the $\mathrm{Ca}$ atom at the $\mathrm{C}-\mathrm{N}$ bond of $\mathrm{HCN} / \mathrm{HNC}$, namely, $\operatorname{HCCaN}\left({ }^{3} \Delta\right)$ and $\mathrm{HNCaC}\left({ }^{3} \Delta\right)$, were also considered. Both isomers are by far less stable than the global minimum c-HCa-NC $\left(115.68 \mathrm{kcal} \mathrm{mol}^{-1}\right.$ and $135.89 \mathrm{kcal} \mathrm{mol}^{-1}$, respectively, at the B2PLYP level of theory).

In order to shed light on the nature of the bond interactions in the most stable isomer, c-HCa-NC, we have employed the QTAIM theory. The molecular graph does not show any ring critical point, and only three critical points between $\mathrm{H}-\mathrm{Ca}, \mathrm{Ca}-$ $\mathrm{N}$, and $\mathrm{N}-\mathrm{C}$ atoms were found. Therefore, this isomer can be described as a $\mathrm{HCa}$ unit $\pi$-bonded to the $\mathrm{CN}$ moiety through the calcium atom, similar to an L-shaped structure.

We have studied the possible interconversion process between the most stable isomers to analyze their stability. Isomer $\mathrm{HCaNC}$ is characterized as a transition state and thus directly isomerizes to the $\mathrm{c}-\mathrm{HCa}-\mathrm{NC}$ minimum. The energy profile for the isomerization reaction $\mathrm{HCaCN} \rightarrow \mathrm{c}-\mathrm{HCa}-\mathrm{NC}$ shows a small barrier of about $0.76 \mathrm{kcal} \mathrm{mol}^{-1}(\sim 382 \mathrm{~K})$ above $\mathrm{HCaCN}$ at the composite level of theory, suggesting that $\mathrm{HCaCN}$ can easily isomerize into c-HCa-NC. Both isomers might coexist if the temperatures of the environment are low.

The analysis of the relative energies and the interconversion reaction predicts two low-lying isomers, c-HCa-NC $\left({ }^{1} \mathrm{~A}^{\prime}\right)$ and hydrocalcium cyanide, $\mathrm{HCaCN}\left({ }^{1} \Sigma\right)$, as possible candidates for experimental or radioastronomical detection. For these isomers, we provide predictions for their molecular structure, thermodynamic stabilities, and vibrational and rotational spectroscopic parameters, which could aid in their identification. Despite the technical difficulties of detecting these new species with currently available telescopes, it is a potentially interesting molecule to search for, so we expect its spectroscopic information is very valuable.

Financial support from the Spanish Ministerio de Economía, Industria y Competitividad (grant AYA2017-87515-P) and the Junta de Castilla y León (grant VA010G18) is gratefully acknowledged.

\section{ORCID iDs}

Pilar Redondo (1) https://orcid.org/0000-0001-7876-4818 Antonio Largo ๑ $\odot$ https://orcid.org/0000-0003-4959-4850 Carmen Barrientos (1) https://orcid.org/0000-0003-0078-7379 


\section{References}

Alessandrini, S., Gauss, J., \& Puzzarini, C. 2018, JChThC, 14, 5360

Asplund, M., Grevesse, N., Sauval, A. J., \& Scott, P. 2009, ARA\&A, 47, 481

Bader, R. F. W. 1990, Atoms in Molecules. A Quantum Theory (Oxford: Clarendon Press),

Becke, A. D. 1988, JChPh, 88, 2547

Cabezas, C., Cernicharo, J., Alonso, J. L., et al. 2013, ApJ, 775, 133

Cernicharo, J., \& Guélin, M. 1987, A\&A, 183, L10

Cernicharo, J., Velilla-Prieto, L., Agúndez, M., et al. 2019, A\&A, 627, L4

Dunbar, R. C., \& Petrie, S. 2002, ApJ, 564, 792

Dunning, T. H. 1989, JChPh, 90, 1007

Frisch, M. J., Trucks, G. W., Schlegel, H. B., et al. 2016, Gaussian 16, Revision B.01 http://www.gaussian.com/gaussian16/

Grimme, S. J. 2006, JChPh, 124, 034108

Gronowski, M., \& Kolos, R. 2013, JPCA, 117, 4455

Guélin, M., Cernicharo, J., Kahane, C., \& Gomez-Gonzalez, J. 1986, A\&A, 157, L17

Guélin, M., Lucas, R., \& Cernicharo, J. 1993, A\&A, 280, L19

Guélin, M., Muller, S., Cernicharo, J., et al. 2000, A\&A, 363, L9

Guélin, M., Muller, S., Cernicharo, J., et al. 2004, A\&A, 426, L49

Hehre, W. J., Radom, L., Schleyer, P. V. P., \& Pople, J. A. 1986, Ab Initio Molecular Orbital Theory (New York: Wiley), 65

Helgaker, T., Klopper, W., Koch, H., \& Noga, J. 1997, JChPh, 106, 9639

Iron, M. A., Oren, M., \& Martin, J. M. L. 2003, MolPh, 101, 1345

Kawaguchi, K., Kagi, E., Hirano, T., et al. 1993, ApJL, 406, L39
Keith, T. A. 2013, AIMAll, version 13.11.04, Professional, TK Gristmill Software, Overland Park, KS, http://aim.tkgristmill.com

Lee, C., Yang, W., \& Parr, R. G. 1988, PhRvB, 37, 785

Matthews, D. A., Cheng, L., Harding, M. E., et al. 2020, JChPh, 152, 214108

Mauron, N. J., \& Huggins, P. J. 2010, A\&A, 513, A31

Mills, I. M. 1972, in Vibration-Rotation Structure in Asymmetric and Symmetric-Top Molecules. In Molecular Spectroscopy: Modern Research, ed. K. N. Rao \& C. W. Mathews (New York: Academic Press), 115

Møller, C., \& Plesset, M. 1934, PhRv, 46, 618

Petrie, S. 1996, MNRAS, 282, 807

Pople, J. A., Head-Gordon, M., Fox, D., Raghavachari, J. K., \& Curtiss, L. A. 1989, JChPh, 90, 5622

Pulliam, R. L., Savage, C., Agúndez, M., et al. 2010, ApJL, 725, L181

Raghavachari, K., Trucks, G. W., Pople, J. A., \& Head-Gordon, M. 1989, CPL, 157,479

Rayón, V. M., Redondo, P., Valdes, H., et al. 2007, JPCA, 111, 6334

Redondo, P., Barrientos, C., \& Largo, A. 2016, ApJ, 828, 45

Redondo, P., Barrientos, C., \& Largo, A. 2019, ApJ, 871, 180

Redondo, P., Largo, A., Vega-Vega, A., \& Barrientos, C. 2015, JChPh, 142 184301

Turner, B. E., Steimle, T. C., \& Meerts, L. 1994, ApJL, 426, L97

Watson, J. K. 1968, MolPh, 15, 479

Woon, D. E., \& Dunning, T. H. 1993, JChPh, 98, 1358

Zack, L. N., Halfen, D. T., \& Ziurys, L. M. 2011, ApJL, 733, L36

Ziurys, L. M., Apponi, A. J., Guélin, M., \& Cernicharo, J. 1995, ApJL, 445, L47

Ziurys, L. M., Savage, C., Highberger, J. L., et al. 2002, ApJL, 564, L45 